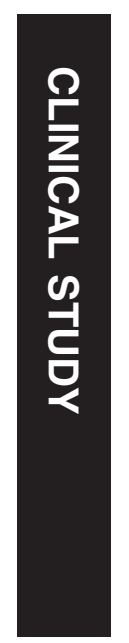

${ }^{1}$ Department of Retina, Narayana Nethralaya, Bangalore, India

${ }^{2}$ Department of Cataract \& Refractive Surgery, Narayana Nethralaya, Bangalore, India

${ }^{3}$ Department of Imaging \& Biomechanics, Narayana Nethralaya, Bangalore, India

${ }^{4}$ Department of Cornea \& Refractive Surgery, Narayana Nethralaya, Bangalore, India

Correspondence: S Dabir, Department of Retina, Narayana Nethralaya, Plot No 121, Chord Road, 1st Block, Rajajinagar, Bangalore 560010, India

Tel: +91 80 66974057;

Fax: +918023377329.

E-mail: supriad@gmail.com

Received: 13 January 2014 Accepted in revised form: 10 August 2014 Published online: 3 October 2014

\title{
Variations in the cone packing density with eccentricity in emmetropes
}

S Dabir ${ }^{1}$, S Mangalesh ${ }^{1}$, KA Kumar ${ }^{1}$, MK Kummelii², A Sinha Roy ${ }^{3}$ and R Shetty ${ }^{4}$

This article has been corrected since Advance Online Publication and a corrigendum is also printed in this issue

\begin{abstract}
Aim To describe the parafoveal cone arrangement in emmetropic subjects and its variations with eccentricity, meridians and change in axial length in Indian eyes. Methods We imaged 25 subjects using compact adaptive optics (AO) retinal camera prototype, the rtx1. Imaging was done at 1, 2, and $3^{\circ}$ eccentricity from the fovea in four meridians: nasal, temporal, superior, and inferior. Results A statistically significant drop in the cone packing density was observed from 2 to $3^{\circ}\left(2^{\circ}\right.$ eccentricity $=25350 / \mathrm{mm}^{2}$ $\left(5300 / \mathrm{mm}^{2}, 8400-34800 / \mathrm{mm}^{2}\right) 3^{\circ}$ eccentricity $=$ $\left.20750 / \mathrm{mm}^{2}\left(6000 \mathrm{~mm}^{2}, 9000-33670 / \mathrm{mm}^{2}\right)\right)$

eliminate spherical aberrations; however, adaptive optics (AO) technology has made it possible to correct the lower and higher order aberrations, allowing better visualization of microscopic structures. ${ }^{1,2}$

Understanding the normal lattice arrangement of photoreceptors aids in detecting early signs of disease and in initial assessment of therapeutic regimes like yellow laser, gene therapy, stem cell therapy, and so on. ${ }^{1}$

The scope of our article is to describe the parafoveal cone arrangement, their variations with eccentricity and interocular symmetry in emmetropic Indian eyes.
\end{abstract} $P<0.05$. The spacing correspondingly increased with increase in distance from the fovea $\left(2^{\circ}\right.$ eccentricity $=6.9 \mu \mathrm{m}(0.70 \mu \mathrm{m}$, $5.95-11.6 \mu \mathrm{m}))$ and $3^{\circ}$ eccentricity $=7.80 \mu \mathrm{m}$ $(1.00 \mu \mathrm{m}, 6.5-13.5 \mu \mathrm{m}) P<0.05$. As the axial length increases, the cone density significantly decreases. Interocular variations were noted. Conclusion With the advent of AO, visualization at the cellular level is now possible. Understanding the photoreceptor mosaic in the parafoveal space in terms of its density, spacing, and arrangement is crucial so as to detect early pathology and intervene appropriately. Newer therapeutic modalitites that are targeted at the cellular level like yellow micropulse laser, stem cells, gene therapy and so on may be better monitored in terms of safety and efficacy.

Eye (2014) 28, 1488-1493; doi:10.1038/eye.2014.229; published online 3 October 2014

\section{Introduction}

Retinal imaging is challenging because of aberrations caused by the tear film, cornea, lens, and internal reflections of eye. Fundus cameras
Materials and methods

Subjects

Twenty-five healthy subjects were included in the prospective study. The study was approved by the institutional review board of the hospital and adhered to the tenets of Helsinki declaration. An informed consent was obtained from all subjects to whom the nature of the study was explained. They underwent a comprehensive ophthalmic examination. Normal eyes were defined as emmetropic subjects or those with best correct visual acuity of 20/20 or better with astigmatism $<2$ D. Subjects with ocular or systemic diseases or previous eye surgery were excluded from the study. All subjects underwent assessment with the Tonoref RKT-7000 autorefractometer, Nidek (Birmingham, AL, USA), non-contact biometry (IOL Master; Carl Zeiss Meditec, Jena, Germany) for axial length, I trace (Tracey Technologies Corp, Houston, TX, USA) for corneal aberrations and spectral-domain optical coherence tomography (Spectralis, Heidelberg, Germany) for central foveal 
thickness. A compact AO retinal camera prototype, the rtx1 (Imagine Eyes, Orsay, France), was used to image the photoreceptor layer. The core components of the apparatus include a Shack-Hartmann wave front sensor (HASO 32-eye; Imagine Optics, Orsay, France), a deformable mirror (MIRAO 52; Imagine Optics), and a low-noise high-resolution camera (Roper Scientific, Tucson, AZ, USA).

\section{Methods}

In this study, the AO imaging sessions were conducted after dilating the pupils with one drop each of $0.5 \%$ tropicamide and $10 \%$ phenylephrine hydrochloride to achieve mid-dilated pupils. Aberrations induced by pupil dilation are negated by the AO system. Stable fixation was maintained by having the patient look at the system's inbuilt target and then as moved by the investigator to pre-determined coordinates. The patient was instructed to fixate at $0,1,2$, and $3^{\circ}$ eccentricity along all the four quadrants: superior, inferior, nasal, and temporal retina. A video (that is, a series of 40 frames; $4{ }^{\circ}$ field size) was captured at each of the above retinal locations. After the acquisition, a program provided by the manufacturer correlated and averaged the captured image frames to produce a final image. ${ }^{3}$

Cone density (cones $/ \mathrm{mm}^{2}$ ) was measured at 1,2 , and $3^{\circ}$ eccentricity along all the four quadrants: superior, inferior, nasal, and temporal retina. There has been no standardized protocol on which areas to image and on the size of the sampling window to choose the region of interest (ROI). The sampling window we chose was $100 \mu \mathrm{m}$ and we placed it at specific coordinates calculated by a pre-fixed formula intentionally avoiding blood vessels. Our rationale was that the size of the sampling window correlates with the size of the retinal area stimulated by a Goldman size III target, so as to be able to correlate the structure and function in the future. Eccentricity was computed as the distance between the center of each window and the foveal center reference point (identified as the point with fixation coordinates: $\left.x=0^{\circ}, y=0^{\circ}\right)$. The images were captured at temporal $\left(-3\right.$ and $\left.0^{\circ}\right)$ superior $\left(0\right.$ and $\left.3^{\circ}\right)$ nasal $\left(3^{\circ}\right.$ and $\left.0^{\circ}\right)$, and inferior $\left(0^{\circ}\right.$ and $\left.-3^{\circ}\right)$ as seen in Figure 1 . The cone counting software AO detect created on MATLAB by Imagine Eyes was used to process the images and calculate the cone density, spacing, and Voronoi. The cone density was verified by three investigators (SD, $\mathrm{SM}$, and $\mathrm{AK}$ ) in order to minimize the possible error in cone identification of the automated software. The inter-observer variability was tested by three observers. The images of all the 25 subjects were analyzed using the AO detect (Imagine Eyes) by each observer separately and the results were noted. The variability in choosing the $\mathrm{ROI}$ and the value of the cone counts was noted between the observers and the closest two values were considered for the interpretation of the results. The subjects were imaged twice at two different time intervals and were analyzed separately. The variation in the cone count was assessed.

\section{Statistical analysis}

Statistical analysis was performed using the SPSS 17 statistics software program (SPSS Inc., Chicago, IL, USA) The Shapiro-Wilk test was applied to note the distribution of data. We applied the Friedman's analysis of variance for the analysis of variation in packing density, spacing, and voronoi at different eccentricities and quadrants and a post hoc test with Bonferroni's correction was applied to the significance level. MannWhitney $U$-test was applied to check for interocular variability in the cone packing density. A simple linear regression was applied to analyze the variation in cone density with axial length.

\section{Results}

The study group comprised of 14 females and 11 males aged between 20 and 40 years as seen in Table 1 . The axial length and spherical equivalent ranged between 21.53 and $24.9 \mathrm{~mm}$ and -0.24 to $+0.13 \mathrm{D}$, respectively. The cone count, spacing, and Voronoi D (50) $P=0.000$ were significantly non-normal in distribution.

Cone packing density, spacing, and Voronoi were analyzed for the four quadrants and the eccentricities separately, as seen in Figure 2. The cone density was found to be significantly different among all the four quadrants (temporal $=25900 / \mathrm{mm}^{2}\left(6100 / \mathrm{mm}^{2}\right.$, $\left.15000-34800 / \mathrm{mm}^{2}\right)$, superior $=21600 / \mathrm{mm}^{2}\left(7400 / \mathrm{mm}^{2}\right.$, $\left.12200-33700 / \mathrm{mm}^{2}\right)$, nasal $=23100 / \mathrm{mm}^{2}\left(4400 / \mathrm{mm}^{2}\right.$, $\left.9000-32700 / \mathrm{mm}^{2}\right)$, and inferior $=20400 / \mathrm{mm}^{2}\left(6900 / \mathrm{mm}^{2}\right.$, $\left.8400-30200 / \mathrm{mm}^{2}\right)$ ) as seen in Table 2. A statistical significance $(P<0.008)$ was found between the orthogonal meridians, that are temporal, nasal $>$ superior, inferior (temporal + nasal $=49000 / \mathrm{mm}^{2}$ $>$ superior + inferior $=42000 / \mathrm{mm}^{2}$ ).

A similar result was observed in the spacing between the cones in the various quadrants (temporal $=6.90 \mu \mathrm{m}$ $(0.80 \mu \mathrm{m}, 5.95-8.8 \mu \mathrm{m})$, superior $=7.60 \mu \mathrm{m}(1.1 \mu \mathrm{m}$, 5.95-13.5 $\mu \mathrm{m})$, nasal $=7.20 \mu \mathrm{m}(0.90 \mu \mathrm{m}, 6.05-11.20 \mu \mathrm{m})$, and inferior $=7.80 \mu \mathrm{m}(0.90 \mu \mathrm{m}, 6.30-11.60 \mu \mathrm{m}))$ as seen in Table 2. Temporal, nasal < superior, inferior (temporal + nasal $=14.1 \mu \mathrm{m}<$ superior + inferior $=15.40 \mu \mathrm{m})$ was found to be statistically significant $(P<0.008)$.

A statistically significant drop in the cone packing density was observed from 2 to $3^{\circ}\left(2^{\circ}\right.$ eccentricity $=$ $\left.25350 / \mathrm{mm}^{2}\left(5300 / \mathrm{mm}^{2}, 8400-34800 / \mathrm{mm}^{2}\right)\right) 3^{\circ}$ eccentricity $=20750 / \mathrm{mm}^{2}\left(6018 / \mathrm{mm}^{2}, 9000-33670 /\right.$ 
a

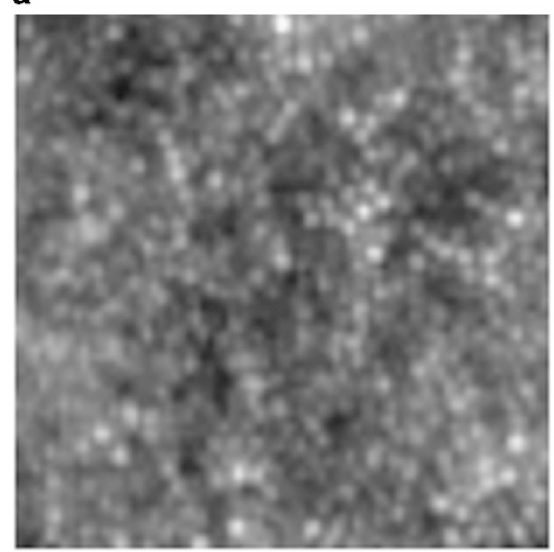

b

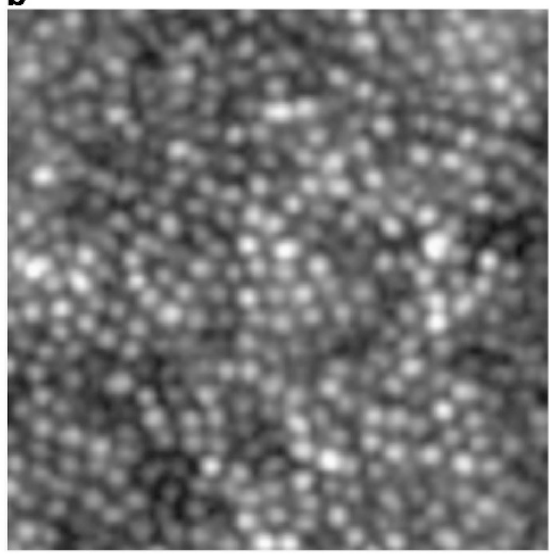

C

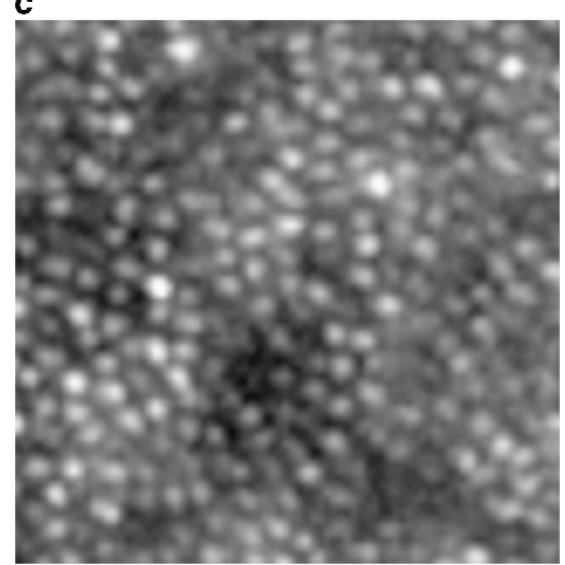

Figure 1 (a-c) Sampling windows showing the cone mosaic at (a) $1^{\circ}$ eccentricity (b) $2^{\circ}$ eccentricity and (c) $3^{\circ}$ eccentricity respectively.

Table 1 Demographics of the study group

\begin{tabular}{lc}
\hline Demographics & Values \\
\hline Total no. of eyes & 25 eyes of 25 volunteers \\
Age group & $20-40$ years \\
Sex (male: female) $(\%)$ & $11: 14(44: 56 \%)$ \\
Axial length (range) & $21.53-24.90 \mathrm{~mm}$ \\
Spherical equivalent (range) & -0.24 to $+0.13 \mathrm{D}$ \\
\hline
\end{tabular}

$\left.\mathrm{mm}^{2}\right) P<0.05$. The spacing correspondingly increased as we moved away from the fovea $\left(2^{\circ}\right.$ eccentricity $=6.9 \mu \mathrm{m}$ $(0.73 \mu \mathrm{m}, 5.95-11.6 \mu \mathrm{m})$ and $3^{\circ}$ eccentricity $=7.80 \mu \mathrm{m}$ $(1.00 \mu \mathrm{m}, 6.5-13.5 \mu \mathrm{m})) P<0.05$.

Voronoi analysis was done to assess whether the cone packing was regular. The number of hexagonal cones were counted and analyzed at the various quadrants and degrees. It was observed that the number of hexagonal cones decreased from 2 to $3^{\circ}\left(2^{\circ}\right.$ eccentricity $=122$ and $3^{\circ}$ eccentricity $=105$ ) and this observation was found to be statistically significant $(P<0.05)$. Difference was also noted in the number of hexagonal cones between the four quadrants $(P<0.05)$. The agreement between the three observers was found to be 0.57 . The difference was found to be in the selection of the ROI between the observers and hence a difference of $\pm 2000 / \mathrm{mm}^{2}$. The subjects were imaged at two different intervals in the same locations to note the variability in the cone count. The difference in the cone counts between the images was found, although the difference was not statistically significant $(P=0.453)$.

We also noted the mean cone count at $1^{\circ}\left(23000 / \mathrm{mm}^{2}\right.$ (2800-32000/ $\left.\mathrm{mm}^{2}\right)$ ). However, counts at or closer than $1^{\circ}$ to the foveal center were not considered for statistical analysis owing to the unreliable values obtained from images near the fovea.

Interocular variability was analyzed at the different retinal eccentricities in all the quadrants as seen in Table 3. No statistically significant difference was found in the cone packing density between the two eyes $(P=0.534)$.

A negative correlation was observed between cone density and axial length at both 2 and $3^{\circ}$, which was found to be statistically significant $\left(R^{2}=0.147 P<0.05\right.$ and $\left.R^{2}=0.236 P<0.05\right)$ as seen in Figure 3. 
a

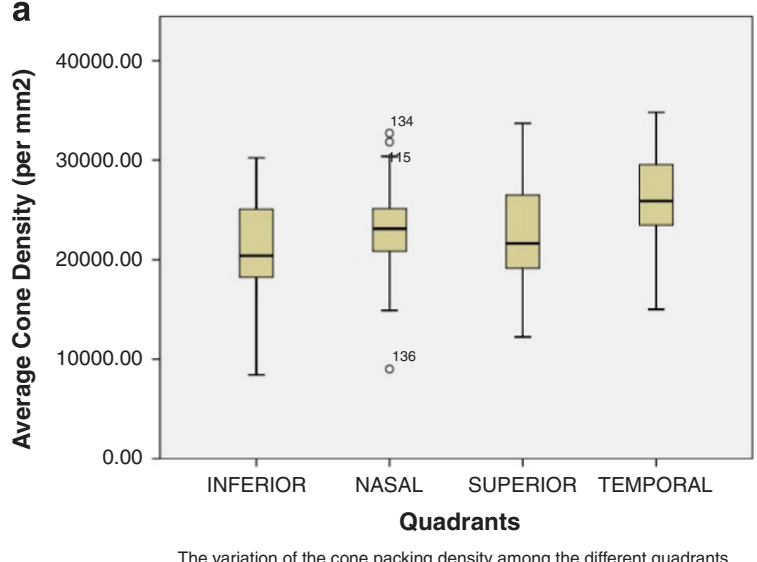

b

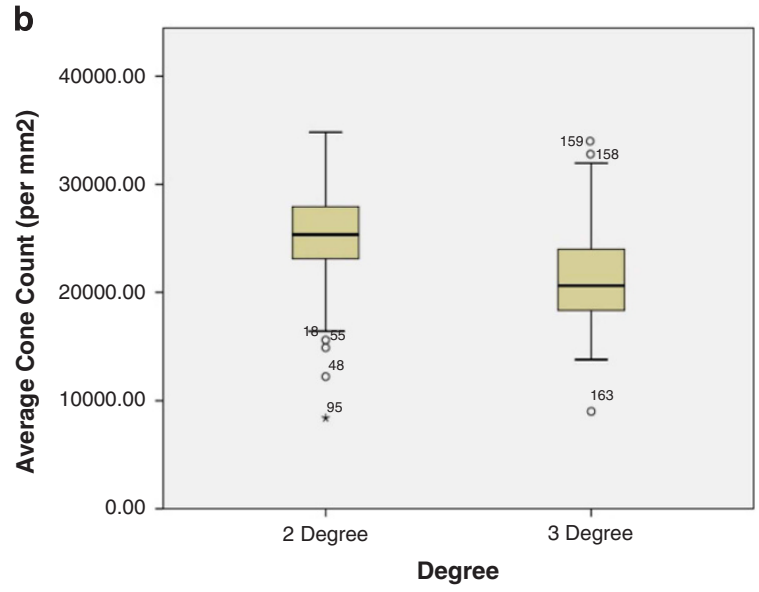

Variation in the Average Cone Count at different eccentricities

C

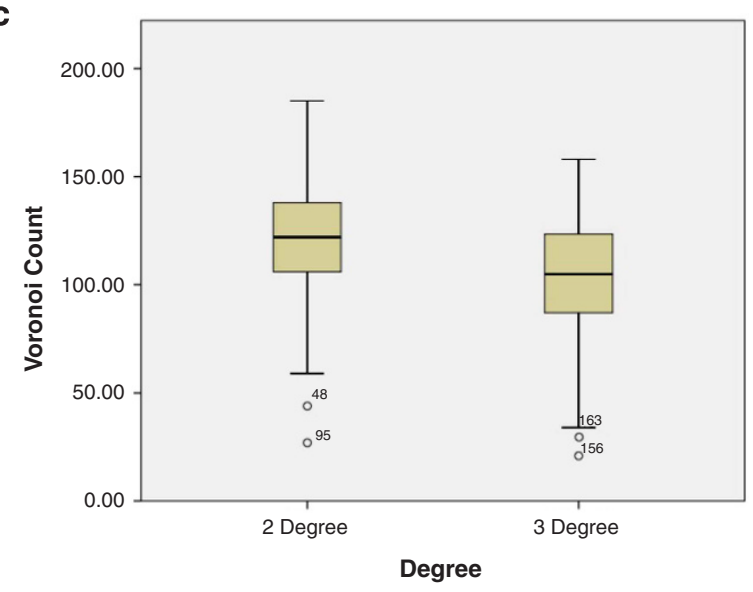

The number of hexagonal cones at different retinal eccentricities

Figure $2(a-c)$. Box plots showing the (a) average cone density distribution in the four quadrants: inferior, nasal, superior, and temporal; (b) average cone density distribution at different retinal eccentricities; (c) Voronoi analyses at different retinal eccentricities.

\section{Discussion}

The average human retina contains 4.6 million cones (4.08-5.29 million). Peak foveal cone density average is
Table 2 Variation in cone packing density and spacing in the four meridians at different retinal eccentricities

\begin{tabular}{lcccccc}
\hline & \multicolumn{2}{c}{ Cone count } & & \multicolumn{2}{c}{ Spacing } & P-value \\
\cline { 2 - 3 } \cline { 5 - 6 } & $\begin{array}{c}2^{\circ} \\
\text { eccentricity }\end{array}$ & $\begin{array}{c}3^{\circ} \\
\text { eccentricity }\end{array}$ & & $\begin{array}{c}2^{\circ} \\
\text { eccentricity }\end{array}$ & $\begin{array}{c}3^{\circ} \\
\text { eccentricity }\end{array}$ & \\
\hline Temporal & 29500 & 24500 & & 6.4 & 8.1 & $0.000(P<0.008)$ \\
Superior & 25700 & 19300 & & 7.0 & 8.0 & $0.000(P<0.008)$ \\
Nasal & 24000 & 23000 & & 7.1 & 7.3 & $0.000(P<0.008)$ \\
Inferior & 24600 & 18400 & & 7.0 & 8.1 & $0.000(P<0.008)$ \\
\hline
\end{tabular}

Table 3 Interocular variability in the cone packing density

\begin{tabular}{lccc}
\hline & $2^{\circ}$ eccentricity & $3^{\circ}$ eccentricity & P-value $^{\mathrm{a}}$ \\
\hline Right eye & 25200 & 21100 & $0.534(P<0.05)$ \\
Left eye & 21400 & 20600 & \\
\hline
\end{tabular}

${ }^{\mathrm{a}}$ Mann-Whitney.

199000 cones $/ \mathrm{mm}^{2}$ and is highly variable between individuals (100000-324000 cones $\left./ \mathrm{mm}^{2}\right){ }^{4}$

Curcio et al (1990) ${ }^{4}$ studied the histology of eight eyes and found that individual variations in the cone density differs with retinal region, highest being near the fovea, least in the mid-periphery and an that it increases again towards the ora serrata.

In our current study, we found the average cone packing densities at 1,2 , and $3^{\circ}$ from the fovea, at $1^{\circ}$, the counts were erroneous owing to the dense packing of the cones and hence the software was unable to count the cones that were $<2 \mu \mathrm{m}$, or the spacing was $<2 \mu \mathrm{m}$, leading to a gross under sampling of the cone density. Hence, they were not utilized for statistical analysis. The counts at 2 and $3^{\circ}$ were $\sim 25350 / \mathrm{mm}^{2}\left(5300 / \mathrm{mm}^{2}, 8400\right.$ $\left.34800 / \mathrm{mm}^{2}\right)$ to $20750 / \mathrm{mm}^{2}\left(6018 / \mathrm{mm}^{2}, 9000-33670 /\right.$ $\left.\mathrm{mm}^{2}\right) \mathrm{P}<0.05$. Park et $a l^{5}$ in their study found that the cone density decreased from 32200 to 11600 cells $/ \mathrm{mm}^{2}$ with retinal eccentricity $(0.5-1.5 \mathrm{~mm}$ from the fovea, $P<0.001)$. Lombardo et $a l^{6}$ found an average decline in cone density from 51000 cones $/ \mathrm{mm}^{2}$ at $250 \mathrm{~mm}$ eccentricity to 14000 cones $/ \mathrm{mm}^{2}$ at $1300 \mathrm{~mm}$ eccentricity along the horizontal meridian.

The numerical variation between the published western literature and our study could be because of various factors such as eyes with different axial lengths, the location of the foveal reference point, the difference in the sampling window size and the non-standardized approach used to calculate cone distribution among the various studies. 5,6 Some have a manual addition to the automated software and a few have used special tools with MATLAB software. ${ }^{5}$

Lombardo et $a l^{1}$ have discussed the various technical differences between the Voronoi maps created using images of the photoreceptor mosaic acquired using 

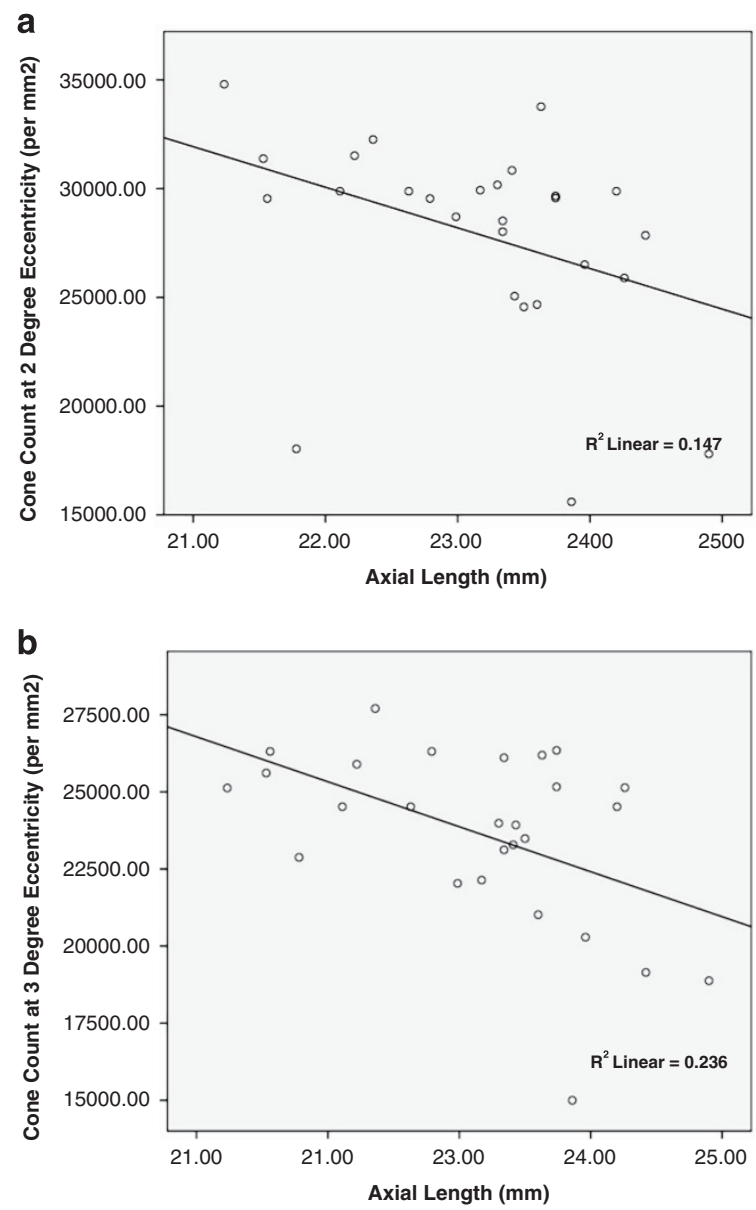

Figure 3 ( $\mathrm{a}$ and $\mathrm{b}$ ). Scatter plot depicting the relation between cone packing density and axial length at (a) $2^{\circ}$ eccentricity and (b) $3^{\circ}$ eccentricity respectively.

different systems, that is, flood-illumination and SLO based. Some of them are due to the differences in the brightness and contrast of the various types of cone mosaics, effect of rods and retinal vessels, and image processing tools.

We have described the distribution of density, spacing and the hexagonal packing arrangement of the cone photoreceptors at different retinal eccentricities across the parafovea in emmetropic young adults. It is essential to establish normative data so as to be able to detect early onset of pathology at cellular level and accordingly intervene early.

Park et $a l^{5}$ compared cone packing density in the four meridians at different eccentricities of $0.5,1$, and $1.5 \mathrm{~mm}$ and found no significant difference, with the exception of $1.0 \mathrm{~mm}$ eccentricity. At $1.0 \mathrm{~mm}$ eccentricity, there were significant differences in the cone packing density between the orthogonal meridians $(P<0.001$; nasal, temporal $>$ superior, inferior). We found the highest density to be in the temporal meridian followed by superior, nasal, and inferior at both the eccentricities of 2 and $3^{\circ}$. Our pattern describes the horizontal packing of cones to be denser than the vertical meridian, as noted by the various histological studies. ${ }^{4,7}$

As cone density decreases with increasing distance from fovea, the spacing will accordingly increase. We found the average spacing to increase from a mean of $7.08 \mu \mathrm{m}$ at $2^{\circ}$ to $7.86 \mu \mathrm{m}$ at $3^{\circ}$. Lombardo $e t \mathrm{al}^{3}$ found the eccentricity to increase from an average of $4.50-8.20 \mu \mathrm{m}$ to $250-1100 \mu \mathrm{m}$, which is similar to ours.

Axial length had a significant role in our study as has been proved by multiple studies ${ }^{5,8,9}$ so far. As the axial length increased, the cone count significantly decreased, following the hypothesis of posterior pole being stretched and the cones getting distributed along a larger surface area. The coefficient of variation between the two eyes of each patient was $30.7 \%$ at $2^{\circ}$ and $21 \%$ at $3^{\circ}$.

The limitation of our study was the absence of a manual addition to the existing automated software to count the missed cones, small sample size, and the data presented was limited to $3^{\circ}$ eccentricity.

\section{Conclusion}

There has been no literature published thus far from the Indian population and our work may help in the development of normative database of individual variations in emmetropic subjects. This allows us to understand early pathology at the cellular level and intervene appropriately. Newer therapeutic modalities that are targeted at the cellular level like yellow micropulse laser, stem cells, gene therapy and so on may be better monitored in terms of safety and efficacy.

\section{References}

1 Lombardo M, Serrao S, Devaney N, Parravano M, Lombardo G. Adaptive optics technology for high-resolution retinal imaging. Sensors 2013; 13(1): 334-366.

2 Chui TY, Song H, Burns S. Adaptive-optics imaging of human cone photoreceptor distribution. J Opt Soc Am A Opt Image Sci Vis 2008; 25(12): 3021-3029.

3 Lombardo M, Serrao S, Ducoli P, Lombardo G. Eccentricity dependent changes of density, spacing and packing arrangement of parafoveal cones. Ophthalmic Physiol Opt 2013; 33(4): 516-526.

4 Curcio C, Sloan K, Kalina R, Hendrickson A. Human photoreceptor topography. J Comp Neurol 1990; 292(4): 497-523.

5 Park S, Chung J, Greenstein V, Tsang S, Chang S. A study of factors affecting the human cone photoreceptor density measured by adaptive optics scanning laser ophthalmoscope. Exp Eye Res 2013; 108: 1-9.

6 Lombardo M, Lombardo G, Schiano Lomoriello D, Ducoli P, Stirpe M, Serrao S. Interocular symmetry of parafoveal photoreceptor cone density distribution. Retina 2013; 33(8): $1640-1649$ 
7 Curcio C, Sloan K. Packing geometry of human cone photoreceptors: variation with eccentricity and evidence for local anisotropy. Vis Neurosci 1992; 9(2): 169-180.

8 Lombardo M, Serrao S, Ducoli P, Lombardo G. Variations in image optical quality of the eye and the sampling limit of resolution of the cone mosaic with axial length in young adults. J Cataract Refract Surg 2012; 38(7): 1147-1155.

9 Chui T, Song H, Burns S. Individual variations in human cone photoreceptor packing density: variations with refractive error. Invest Ophthalmol Vis Sci 2008; 49(10): 4679-4687. 